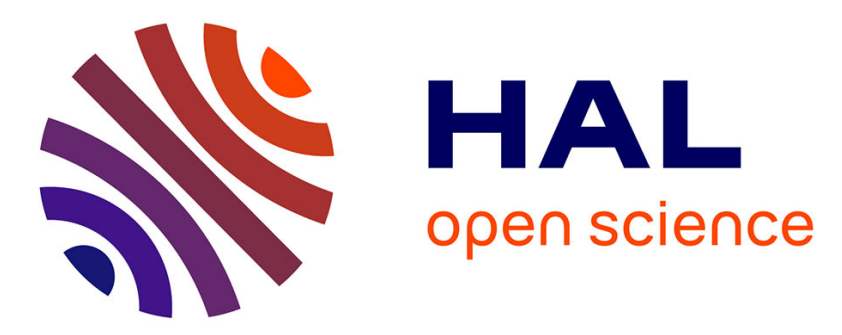

\title{
Modular Assembly of Multimodal Imaging Agents through an Inverse Electron Demand Diels-Alder Reaction
}

\author{
Coline Canovas, Mathieu Moreau, Jean-Marc Vrigneaud, Pierre-Simon \\ Bellaye, Bertrand Collin, Franck Denat, Victor Goncalves
}

\section{To cite this version:}

Coline Canovas, Mathieu Moreau, Jean-Marc Vrigneaud, Pierre-Simon Bellaye, Bertrand Collin, et al.. Modular Assembly of Multimodal Imaging Agents through an Inverse Electron Demand Diels-Alder Reaction. Bioconjugate Chemistry, 2019, 30 (3), pp.888-897. 10.1021/acs.bioconjchem.9b00017. hal-03470979

\section{HAL Id: hal-03470979 \\ https://hal.science/hal-03470979}

Submitted on 8 Dec 2021

HAL is a multi-disciplinary open access archive for the deposit and dissemination of scientific research documents, whether they are published or not. The documents may come from teaching and research institutions in France or abroad, or from public or private research centers.
L'archive ouverte pluridisciplinaire HAL, est destinée au dépôt et à la diffusion de documents scientifiques de niveau recherche, publiés ou non, émanant des établissements d'enseignement et de recherche français ou étrangers, des laboratoires publics ou privés. 
Modular assembly of multimodal imaging agents through an inverse electron demand Diels-Alder reaction

Coline Canovas, ${ }^{\dagger}$ Mathieu Moreau, ${ }^{\dagger}$ Jean-Marc Vrigneaud, ${ }^{\ddagger}$ Pierre-Simon Bellaye,${ }^{\ddagger}$ Bertrand Collin, ${ }^{\dagger, \dagger}$ Franck Denat, ${ }^{\dagger}$ and Victor Goncalves ${ }^{\dagger, *}$

† Institut de Chimie Moléculaire de l'Université de Bourgogne, UMR6302, CNRS, Université Bourgogne Franche-Comté, 9 avenue Alain Savary, 21000, Dijon (France)

$\$$ Georges-François LECLERC Cancer Center - UNICANCER, 1 rue Pr Marion, 21079, Dijon (France)

* Correspondence should be addressed to V. Goncalves (victor.goncalves@u-bourgogne.fr; Phone: +33 (0)3 803960 88; Fax: +33 (0)3 803961 17)

\begin{abstract}
The combination of two imaging probes on a same biomolecule gives access to targeted bimodal imaging agents that can provide more accurate diagnosis, complementary information, or that may be used in different applications, such as PET imaging and fluorescence imagingassisted surgery. In this study, we demonstrate that dichlorotetrazine, a small, commercially available compound, can be used as a modular platform to easily assemble various imaging probes. Doubly-labeled tetrazines can then be conjugated to a protein through a biorthogonal IEDDA reaction. A series of difunctionalized tetrazine compounds containing various chelating agents and fluorescent dyes was synthesized. As a proof of concept, one of these bimodal probes was conjugated to trastuzumab, previously modified with a constrained alkyne group, and the resulting dual-labeled antibody was evaluated in a mice model, bearing a HER2-positive tumor. A significant uptake into tumor tissues was observed by both SPECT-CT and fluorescence imaging, and confirmed ex-vivo in biodistribution studies.
\end{abstract}

\title{
KEYWORDS
}

Tetrazine; Site-specific; bimodal; SPECT-CT; fluorescence; trastuzumab 


\section{INTRODUCTION}

Molecular imaging is a powerful tool that allows the non-invasive visualization of physiological processes that occur in living organisms. In particular, actively targeted molecular imaging agents, which associate an imaging probe with a vector able to recognize biological targets of interest, have proven to be extremely effective in the rapid and accurate diagnosis of many diseases. ${ }^{1}$ Two or more imaging techniques can eventually be combined together to perform multimodal imaging examinations, that generate simultaneously complementary data, facilitating image interpretation and thus increasing the reliability of the diagnosis. ${ }^{2}$ For instance, the combination of fluorescence and nuclear imaging, although still in its infancy in clinical setting, appears as extremely promising in oncology. ${ }^{3,4}$ Indeed, both techniques are extremely sensitive and thus very low concentrations of hybrid imaging agent can be visualized indifferently according to one or the other of the techniques. A remarkable application of bimodal imaging is the combination of i) the diagnostic properties of nuclear scintigraphy techniques for pre-operative identification of cancerous areas and surgery planning, with ii) the potential of fluorescence-guided surgery to improve the quality of tumor resection. ${ }^{5,6}$ The emission of gamma rays by radionuclides can also be advantageously used to perform radioguided cancer surgery using gamma probes, which allow the detection and resection of very small lesions, even when they are covered by healthy tissue. ${ }^{7}$

Unfortunately, the synthesis of protein-based multimodal molecular imaging agents is still challenging. ${ }^{8,9}$ Indeed, the bioconjugation step can be particularly complex and difficult to control, and a simple modification of protein's tertiary structure can considerably alter its recognition properties. Furthermore, the introduction of an excessive number of hydrophobic molecules can make a protein unstable and generate aggregation problems. ${ }^{10}$ Pharmacokinetics can also be significantly modified compared to that of the native biovector. ${ }^{11}$ These problems do not only concern the modification of small proteins, such as Affibodies or antibody fragments, but also affect full-length antibodies. The random labeling of proteins with two different payloads only reinforces this phenomenom. Thus, in recent years, regiospecific conjugation techniques have been at the heart of many studies and several reviews have dealt with this subject in detail. ${ }^{12-15}$ Site-specific conjugation makes it possible to control both the number of imaging probes introduced and their location on the protein. Being able to provide fully-defined, homogeneous bioconjugates, with higher reproducibility from batch to batch, also facilitates their approval by regulatory agencies. In addition, for doubly-labeled proteins, it allows full control of the ratio between the two imaging probes. Among the different strategies 
developed, the incorporation of unnatural amino acids (uAAs) by extension of the genetic code is probably one of the most powerful techniques. It makes possible the introduction of various bioorthogonal anchors, which then allow the site-specific coupling of imaging probes by means of highly chemoselective click reactions. Among them, the Diels-Alder reaction with inverse electron-demand (IEDDA) tends to outperform other click chemistry reactions in terms of kinetics, orthogonality and biocompatibility. ${ }^{18,19}$ IEDDA reaction relies on a [4+2] cycloaddition that typically involves a 1,2,4,5-tetrazine heterocycle, and a strained alkene or alkyne that acts as a dienophile. ${ }^{20-22}$ There is a wide variety of uAAs with constrained alkene, alkyne, or 1,2,4,5-tetrazine function on their side chain that can be incorporated into recombinant proteins. ${ }^{19}$

An effective method for the synthesis of clickable imaging agents suited to IEDDA reaction consists in the construction of the tetrazine cycle from a nitrile-functionalized imaging probe. Using this approach, Weissleder et al. were able to develop the first, and sole example to date, of a clickable bimodal optical/nuclear probe based on a reactive tetrazine. ${ }^{23}$ Starting from $\mathrm{p}$ cyanophenyl-BODIPY, they formed the tetrazine ring and subsequently introduced deferoxamine, a linear chelator used for the complexation of ${ }^{89} \mathrm{Zr}$, on the BODIPY boron atom. This molecule was clicked to an antibody carrying a trans-cyclooctene group to afford a targeted PET/fluorescent imaging agent. Despite its efficiency, this synthetic strategy suffers from its length, limited modularity, and is not necessarily easy to transpose to other families of imaging agents.

In search of a minimalist platform that would allow the straightforward introduction of various imaging probes, and their site-specific coupling to proteins by IEDDA reaction, we were interested in the chemistry of 3,6-dicholorotetrazine. Indeed, it has been shown that dichlorotetrazine can be substituted by various nucleophiles, such as amines, alcohols or thiols, via the aromatic nucleophilic substitution of its chlorine atoms, and that the tetrazine ring can participate in an IEDDA reaction with alkenes or alkynes. ${ }^{24-28}$ This reactivity was leveraged to introduce two imaging probes on a protein, the bioconjugation being done by means of a nucleophilic aromatic substitution between the chlorotetrazine and the thiol group of a cysteinyl residue in the protein. ${ }^{29}$

In this study, we decided to investigate whether the dichlorotetrazine could be used as a ternary platform to achieve the dual, site-specific labeling of proteins by IEDDA reaction. We show that a series of bimodal imaging probes, incorporating a chelator for radiometals and a fluorescent dye, can be readily assembled on the tetrazine platform through successive 
nucleophilic aromatic substitution $\left(\mathrm{S}_{\mathrm{N}} \mathrm{Ar}\right)$ reactions. These bimodal probes can later on react selectively with a protein carrying a reactive alkene or alkyne group through a IEDDA reaction to afford a doubly-modified protein (Figure 1). This strategy is exemplified with the preparation of a dual-labeled antibody and its evaluation as a bimodal imaging agent on a mice model bearing a HER2-positive tumor by SPECT-CT and near-infrared fluorescence imaging.

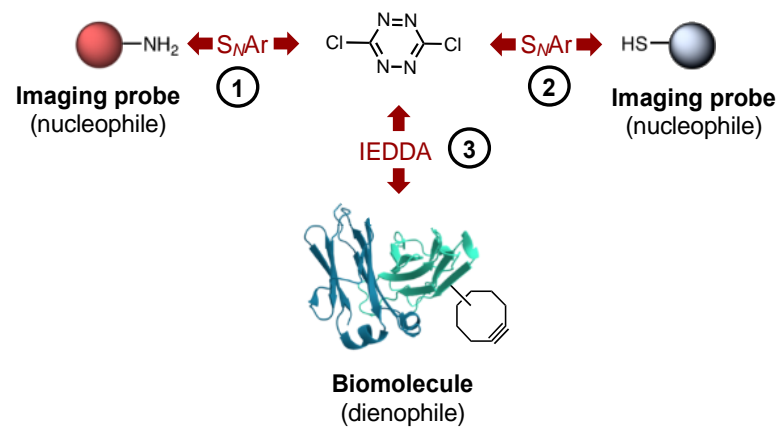

Figure 1. General strategy for the site-specific dual-labeling of proteins with dichlorotetrazine through a IEDDA reaction. 


\section{RESULTS AND DISCUSSION}

Synthesis of a series of bimodal tetrazines. The choice of appropriate nucleophiles is essential to perform the double substitution of the dichlorotetrazine. Generally, the substitution of only one of the chlorine atoms in dichlorotetrazine can be easily carried out at room temperature by amines, alcohols or thiols. ${ }^{24}$ On the other hand, as shown in several studies, the substitution of the second chlorine atom is more difficult and requires the use of stronger nucleophiles. ${ }^{26}$ This is caused by the increase in tetrazine's electronic density induced by the introduction of the first nucleophile. However, this decrease in reactivity of the monosubstituted chlorotetrazine with regard to the second substitution is actually an advantage, when dichlorotetrazine is used as a platform, since it generates a certain degree of orthogonality between the two $S_{N} A r$ reactions. In order to facilitate the synthesis of multimodal imaging agents, we decided to carry out the first $\mathrm{S}_{\mathrm{N}} \mathrm{Ar}$ with an amine nucleophile. Indeed, imaging probes, and other payloads such as drugs, functionalized by an amine group, are frequently used and are therefore often commercially available.

The first functionalization of dichlorotetrazine was performed with macrocyclic chelators functionalized by primary amines: DOTAGA-NH 2 and (R)-NODAGA-NH2 (Figure 2b). The DOTAGA macrocycle is a bifunctional derivative of 1,4,7,10-tetraazacyclododecane-1,4,7,10tetraacetic acid, also called DOTA, the workhorse of nuclear medicine with radiometal isotopes. It is able to chelate efficiently different radiometals such as indium-111 for SPECT imaging, yttrium-86, gallium-68 and copper-64 for PET imaging, lutetium-177 and yttrium-90 for radiotherapy applications. $^{30}$ (R)-NODAGA is a bifunctional derivative of 1,4,7triazacyclonane-1,4,7-triacetic acid, also known as NOTA, which has proven superior to DOTA for the complexation of copper-64 and gallium-68 for PET imaging, or gallium-67 for SPECT. ${ }^{31,32}$ The low solubility of macrocyclic compounds in organic solvents led us to carry out the reaction in a mixture of borate buffer $\mathrm{pH} 8(0.5 \mathrm{M})$ and acetonitrile $(80: 20, v / v)$. The compounds DOTAGA-NH-Tz-Cl 1 and (R)-NODAGA-NH 2 were obtained in a few hours at room temperature. ${ }^{29}$ 


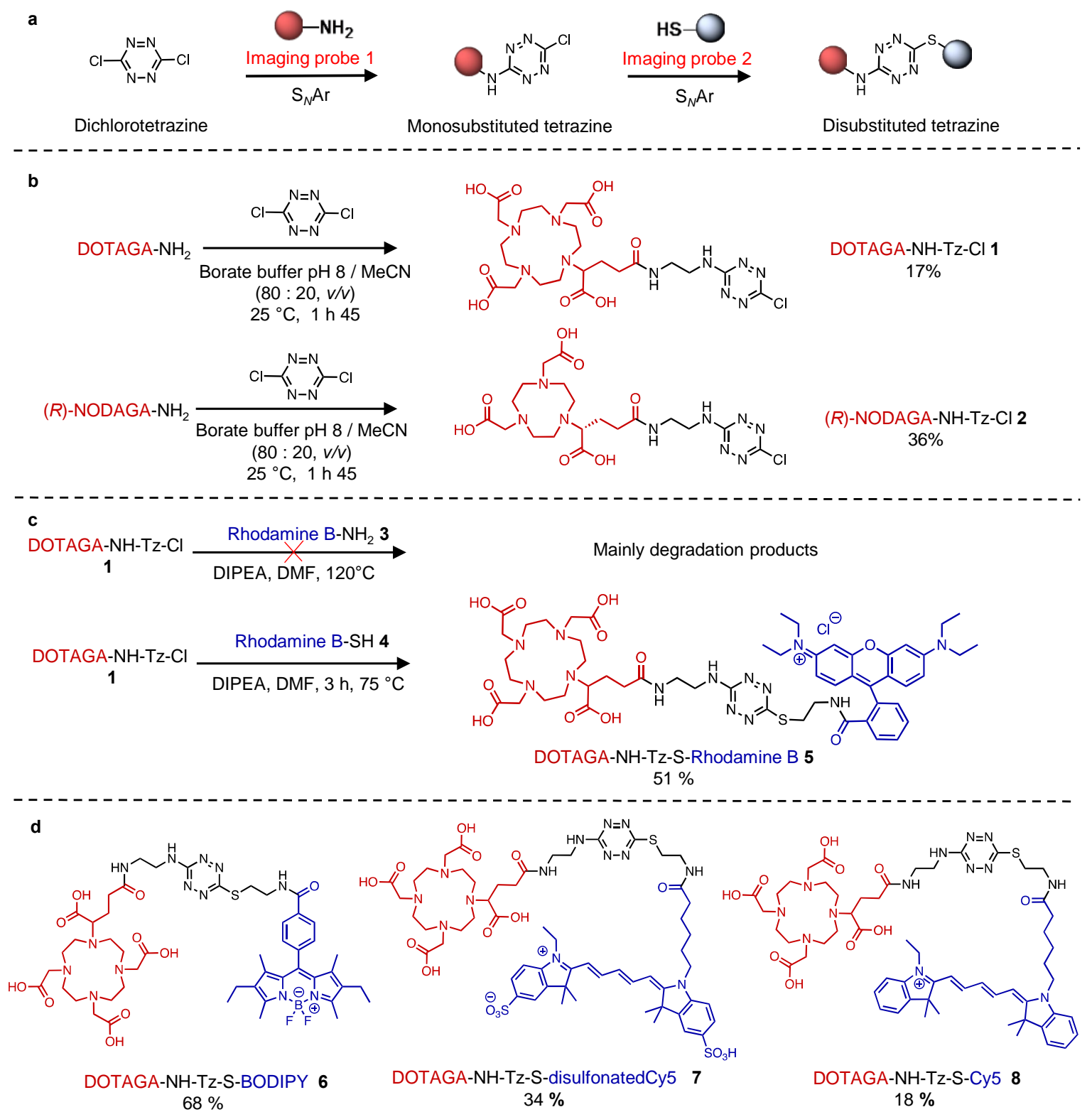

Figure 2. Synthesis of bimodal imaging probes based on the tetrazine platform. a. General approach for the two-step synthesis of clickable bimodal imaging probes. b. Synthesis of monosubstituted chlorotetrazines $\mathbf{1}$ and 2. c. Reactivity of the monosubstituted tetrazine $\mathbf{1}$ with regard to amine and thiol derivatives. d. Structure of different disubstituted tetrazines and corresponding yields.

In order to prepare a bimodal nuclear/optical imaging agent, we tried to substitute the second chlorine atom with a fluorescent agent. Rhodamine B, a fluorophore emitting in the visible region at ca. $550 \mathrm{~nm}$, was used to perform preliminary tests and determine the most appropriate nucleophile for this step. Nucleophilic derivatives, amine (3) and thiol (4), of rhodamine B were synthesized in good yields by peptide coupling of ethylene diamine or cysteamine 
(Supplementary information). Compounds $\mathbf{3}$ and $\mathbf{4}$ were then exposed to the DOTAGAmonosubstituted chlorotetrazine 1 (Figure 2c). As expected, the second $\mathrm{S}_{\mathrm{N}} \mathrm{Ar}$ by an amine nucleophile proved to be difficult and could not be performed under mild conditions. Heating the reaction medium for several hours at $120^{\circ} \mathrm{C}$ gave a considerable proportion of degradation products, which led us to quickly abandon this approach. However, the second substitution was carried out in 3 hours at $75^{\circ} \mathrm{C}$ by the more nucleophilic Rhodamine B-SH compound 4 . The corresponding disubstituted tetrazine $\mathbf{5}$ was isolated by RP-HPLC with a yield of $51 \%$.

To assess the versatility of the strategy, we synthesized, in addition to $\mathbf{5}$, three examples of bifunctionalized tetrazines, based on different fluorophores including a compound from the boron-dipyromethene family (6) and two non-symmetric cyanines 5.0 with (7) or without (8) water-solubilizing sulfonate groups, in moderate to good yields (Figure 2d).

Bioconjugation to trastuzumab through a IEDDA reaction. The doubly substituted tetrazine 7, bearing a DOTAGA macrocycle and a disulfonated cyanine 5 probe, was selected for further study. DOTAGA allows the complexation of indium-111, a $\gamma$-emitter with a relatively long half-life (2.8 days). ${ }^{33}$ This macrocycle is therefore suitable for tumor targeting by imaging agents with slow pharmacokinetics such as antibodies. The fluorophore was chosen for its attractive spectral properties in the near-infrared I region. The presence of sulfonate groups is also a significant asset to facilitate the bioconjugation step, because it limits the aggregation of the conjugate and increases its water solubility.

Before starting the bioconjugation step, we decided to evaluate the stability of $\mathbf{7}$ in pure water. The proportion of intact compound was measured by integrating the RP-HPLC chromatogram at the $214 \mathrm{~nm}$ detection wavelength (Supplementary Fig. S30). The disubstituted tetrazine was found to be relatively stable, with $80 \%$ of the compound intact after one night of incubation at room temperature. The slight degradation observed should be easily compensated by the use of an excess of disubstituted tetrazine during bioconjugation.

Trastuzumab was chosen as a model to evaluate the feasibility of the bioconjugation step. This monoclonal antibody has a strong affinity for the human epidermal growth factor receptor 2 (HER2), which is overexpressed by certain mammary cancer cells. ${ }^{34}$ Trastuzumab is currently used clinically to treat breast cancer and is also known for its effectiveness as a targeting vector to prepare molecular imaging agents. ${ }^{33}$ In order to obtain a protein capable of reacting with the tetrazine by IEDDA reaction, we inserted a bicyclo[6.1.0]nonyne (BCN) function on 
trastuzumab. Although this clickable function can be introduced in a site-specific way, ${ }^{16,35}$ it was deemed sufficient to perform the random labeling of the amine functions of trastuzumab with BCN-NHS to establish the feasibility of the bioconjugation by IEDDA reaction (Figure 3). Analysis of the modified antibody 9 by MALDI-TOF mass spectrometry revealed an approximate degree of labeling (DOL) of 3.3 BCN groups per trastuzumab.

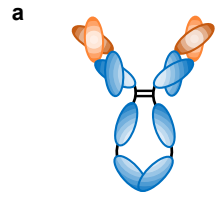

Trastuzumab

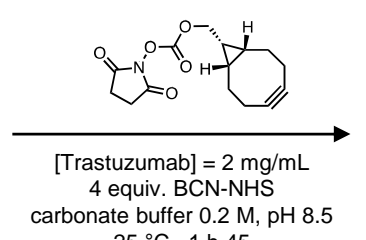

$25^{\circ} \mathrm{C}, 1 \mathrm{~h} 45$

b

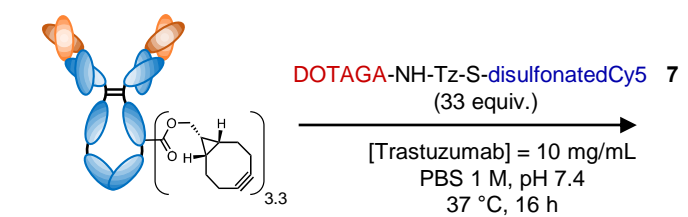

Trastuzumab-BCN 9
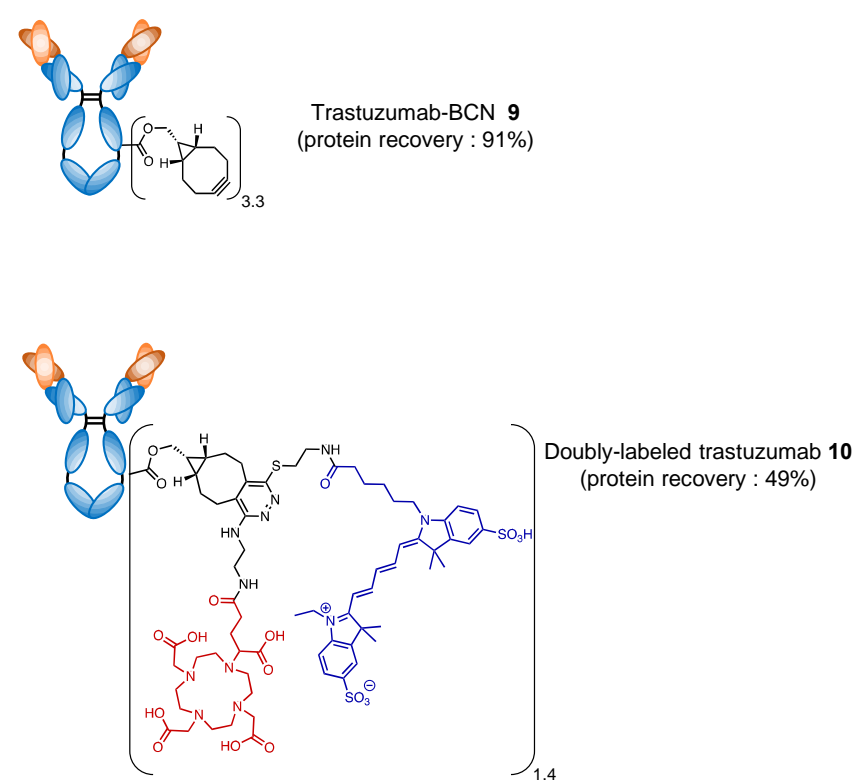

Figure 3. Synthesis of doubly-labeled trastuzumab 10. a. Preparation of trastuzumab-BCN by random labeling of lysine residues; $b$. Conjugation of 9 with the bimodal probe DOTAGA$\mathrm{NH}-\mathrm{Tz}-\mathrm{S}$-disulfonatedCy 7 by IEDDA reaction. The degrees of labeling were determined by mass spectrometry or UV-Vis spectroscopy.

The double-modification of the antibody was performed by mixing 33 equivalents of disubstituted tetrazine 7 with trastuzumab-BCN 9 (Figure 3b). As mentioned above, the introduction of nitrogen and sulfur heteroatoms electronically enriches the tetrazine heterocycle and thus reduces its reactivity. The conjugation of the bimodal probe to the antibody was therefore quite slow and in order to increase the conversion, it was conducted overnight at $37^{\circ} \mathrm{C}$. The removal of the excess of $\mathbf{9}$ by ultrafiltration was not possible due to the presence of the fluorophore, which binds to filter membranes through non-specific interactions. To avoid these problems, the doubly modified antibody $\mathbf{1 0}$ was purified by FPLC on a desalting column, and eluted with an ammonium acetate buffer $(0.1 \mathrm{M}, \mathrm{pH} 5.8)$ that will be used during the radiolabeling step. 10 was isolated with $49 \%$ protein recovery. 
The mass spectrum analysis of the bioconjugate by MALDI-TOF did not allow the precise calculation of the DOL. However, it could be quantified from the UV-visible spectrum of the conjugate, by comparing the absorption of the cyanine dye, at $648 \mathrm{~nm}$, with the absorption of the protein at $280 \mathrm{~nm}$ (a correction factor was introduced to account for the contribution of the fluorophore absorption at $280 \mathrm{~nm}$ ). An average DOL of 1.4 bimodal tetrazine per antibody was obtained (Figure 4). These analyses showed that less than half of the bicyclo[6.1.0]nonyne groups introduced on the antibody had actually reacted. The lack of reactivity of some BCN groups can be probably attributed to their lower accessibility on the protein, as the IEDDA reaction is known to be sensitive to steric hindrance. ${ }^{36}$
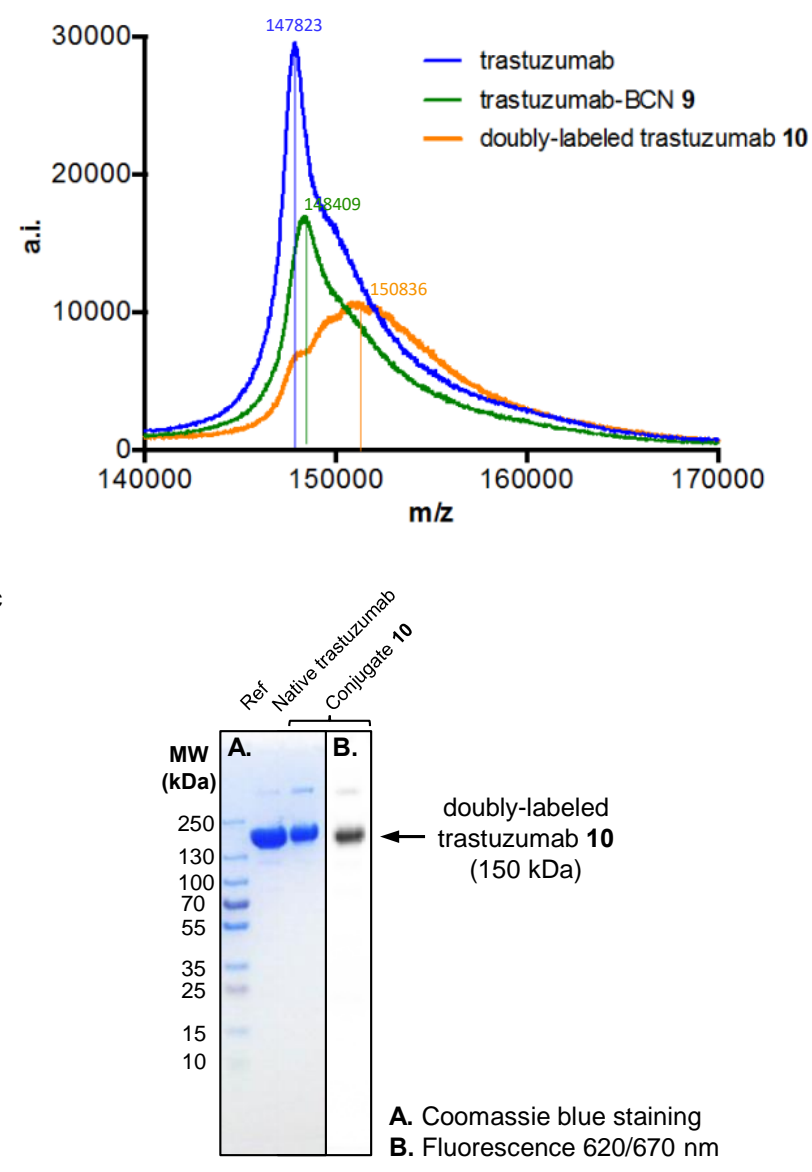

b

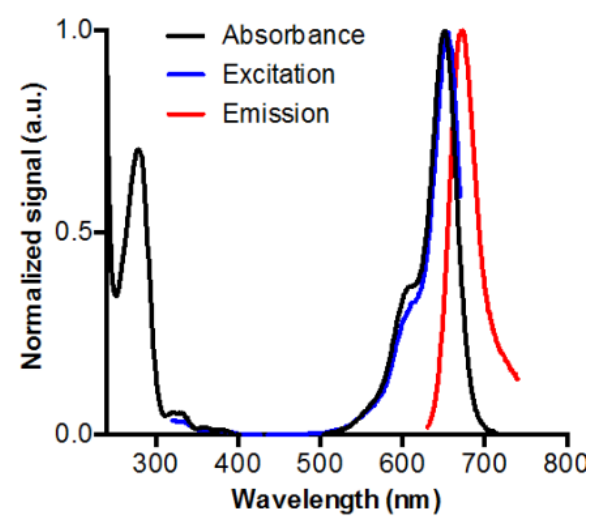

d

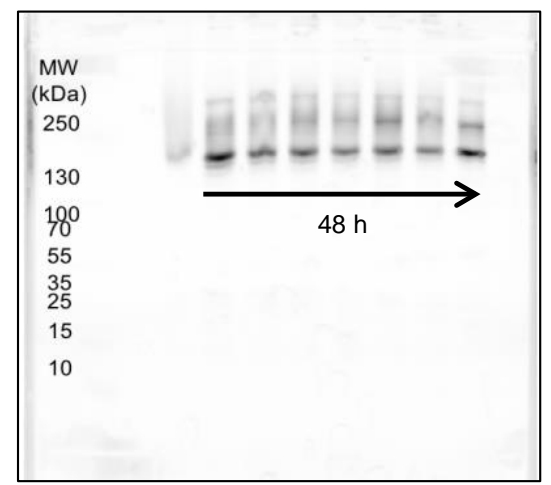

Figure 4. Characterization and stability of the doubly-modified trastuzumab 10. a. MALDI-TOF mass spectra of native trastuzumab, trastuzumab modified with BCN 9 and doubly-modified trastuzumab 10. a.i. : native intensity; b. Absorption, excitation and emission spectra of compound $\mathbf{1 0}$ in ammonium acetate buffer $(0.1 \mathrm{M}, \mathrm{pH} 5.8)$, at $25^{\circ} \mathrm{C}$. a.u. : arbitrary units; c. SDS-PAGE analysis of native trastuzumab and 10, with Coomassie blue revelation and 
fluorescence acquisition. Uncropped images of the gel are available as Supplementary Fig. S34; d. SDS-PAGE analysis of the conjugate $\mathbf{1 0}$ after incubation in human plasma at $37^{\circ} \mathrm{C}$ for 0,1 , 2, 4, 8, 24 and $48 \mathrm{~h}$ (fluorescent signal). The corresponding Coomassie blue gel is available as Supplementary Fig. S35.

The compound was also evaluated by polyacrylamide gel electrophoresis in the presence of sodium dodecyl sulfate (SDS-PAGE) (Figure 4c). Fluorescence analysis of the gel showed the absence of a signal corresponding to the free cyanine, which proves the covalent nature of the link between the antibody and the fluorescent probe. This is a parameter that we felt it was important to verify because near-infrared fluorescent probes are known for their ability to adsorb strongly to proteins in a non-specific way. ${ }^{37}$

Finally, the stability of the conjugate was evaluated after incubation at $37^{\circ} \mathrm{C}$ in human plasma. Samples were taken at different times and analyzed by SDS-PAGE (Figure 4d). The absence of release of free fluorophore showed that the compound was stable up to $48 \mathrm{~h}$ in these assay conditions.

Radiolabeling and SPECT-CT/Fluorescence imaging of the bioconjugate. Radiolabeling of the compound 10 with indium-111 afforded the conjugate 11. Briefly, $\left[{ }^{111} \mathrm{In}\right]-\mathrm{InCl}_{3}$, in solution in $0.05 \mathrm{M} \mathrm{HCl}$, was added to $\mathbf{1 0}$ to obtain a proportion of $600 \mathrm{MBq}$ per mg of conjugate and the solution was buffered to $0.1 \mathrm{M} \mathrm{AcONH}_{4}$. After $1 \mathrm{~h}$, the reaction was quenched by adding an excess of EDTA. After purification, $310 \mu \mathrm{g}$ of compound $\mathbf{1 1}$ were thus isolated with a radiochemical purity of $99 \%$ and a specific activity of $366 \mathrm{MBq} / \mathrm{mg}$ (Supplementary information).

The targeting properties of conjugate $\mathbf{1 1}$ were then studied in a model of human breast adenocarcinoma (BT-474 cells), HER2-positive, xenografted subcutaneously on BALB/c nude mice. A first group of mice (Group $1, \mathrm{n}=4$ ) was injected with approximately $24 \mu \mathrm{g}(8.7 \mathrm{MBq})$ of compound 11 per mouse and a second group of mice (Group 2, $\mathrm{n}=3$ ) was used to perform blocking experiments. Blocking experiments were done by co-injecting approximately $20 \mu \mathrm{g}$ (6.9 MBq) of compound 11 and 100 equivalents of native trastuzumab per mouse. Images of live mice were taken 24 hours p.i. using both modalities (SPECT-CT and fluorescence).

The images obtained at $24 \mathrm{~h}$ p.i., by SPECT-CT showed good tumor-to-background ratios. The blocking experiment confirmed the specificity of the binding on HER2-positive tumors (Figure 5a). Fluorescence imaging showed a similar profile, with easily detectable tumors (Figure 5b). 

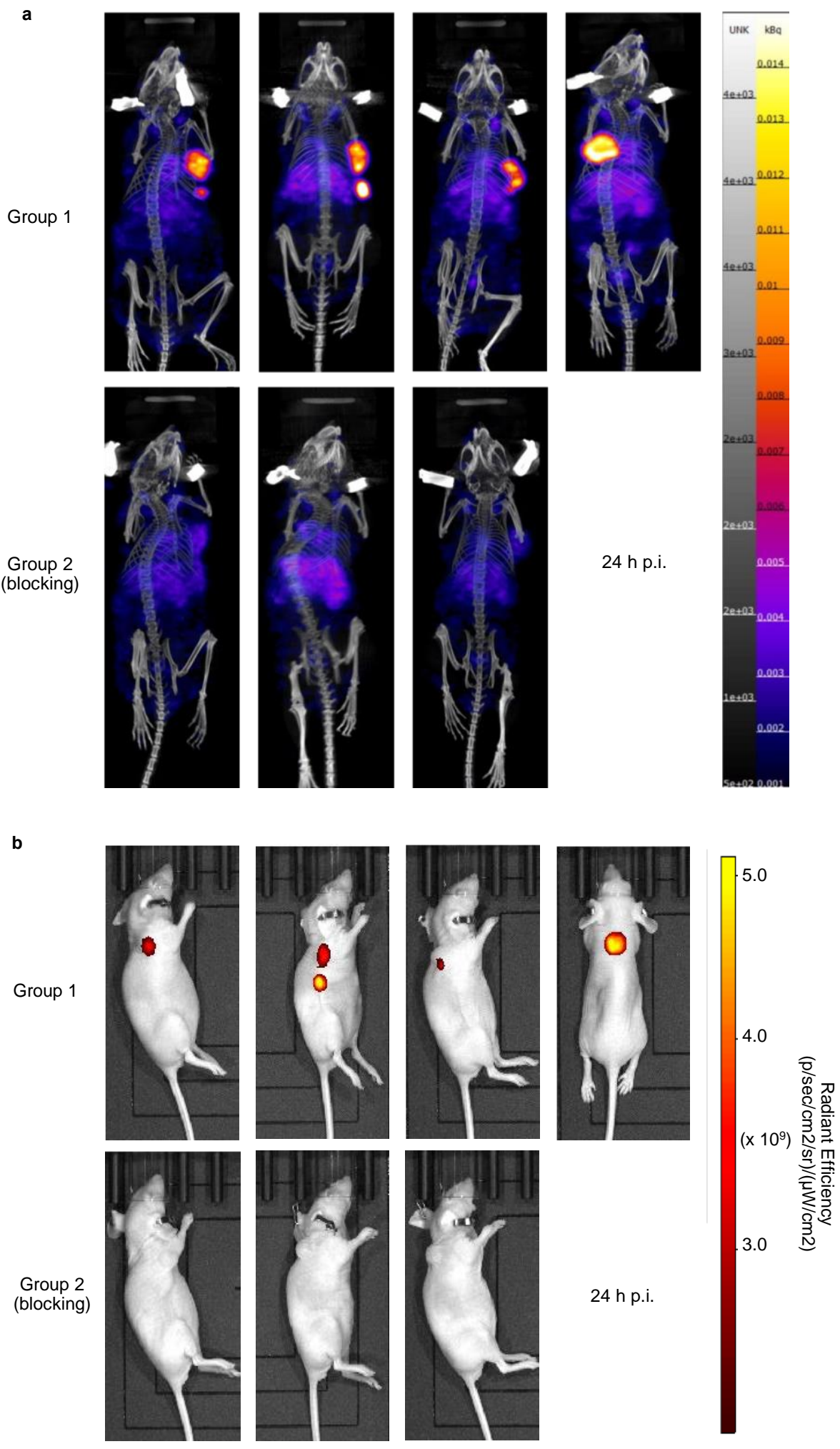

Figure 5. In vivo distribution of the bioconjugate 11 by SPECT-CT and fluorescence imaging. a. SPECT-CT imaging in living mice at $24 \mathrm{~h}$ p.i.; SPECT maximum intensity 
projections were fused to CT images; $b$. Images of the biodistribution by the measurement of fluorescence (filter mode Ex./Em. 620/670 nm) in living mice at $24 \mathrm{~h}$ p.i.; the fluorescence signal, displayed as radiant efficiency $\left(\left[\mathrm{p} / \mathrm{s} / \mathrm{cm}^{2} / \mathrm{sr}\right] /\left[\mu \mathrm{W} / \mathrm{cm}^{2}\right]\right)$, is superimposed to white light images; the positions were chosen for the best visibility of the tumor.

The mice were then euthanized and the biodistribution of compound $\mathbf{1 1}$ was measured by gamma counting of the various organs collected (Figure 6). Measurement of the radioactive signal ex vivo confirmed a strong accumulation of the compound in tumor tissues. The tumor/muscle and tumor/blood ratios reached values of 28.6 and 3.1 respectively. However, the relatively low tumor-to-blood ratio value suggests that the conjugate was still circulating in the bloodstream after 24 hours, which could probably be improved by measuring biodistribution at longer times p.i. (48 or $72 \mathrm{~h})$.

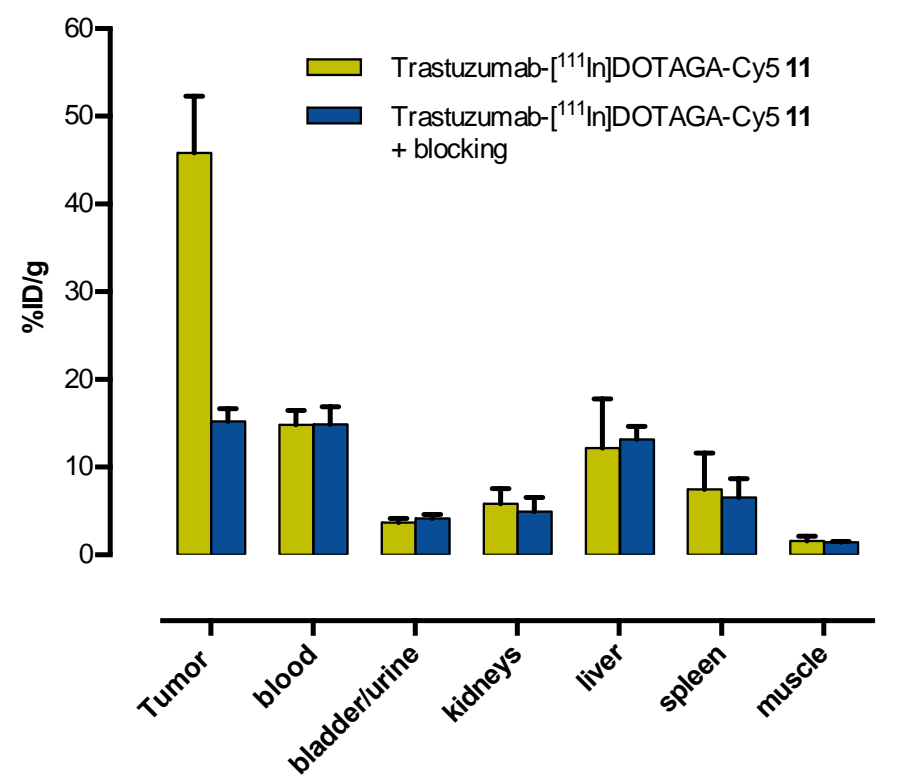

Figure 6. Biodistribution data. Values are expressed in percentage of injected dose per gram (\%ID/g) for each collected organ (tumor, liver, kidneys, blood, muscle, bladder/urine, spleen). Data are expressed as means and SD for the two groups with $n=4$ for group 1 and $n=3$ for group 2. Individual data are available in Supplementary Fig. S38.

Biodistribution was also calculated from fluorescence images (Supplementary Fig. S40). However, these data should be taken with caution as the fluorescent signal is difficult to 
quantify due to its high dependence on various phenomena such as tissue diffusion, attenuation and autofluorescence.

The concordance between the biodistributions observed according to the two imaging modalities (gamma counting and fluorescence) shows that radiolabeled compound $\mathbf{1 1}$ is sufficiently stable in vivo. This preclinical study thus confirmed the viability of our bioconjugation strategy for small animal diagnostic applications. 


\section{CONCLUSION}

In summary, this study presents a new strategy for the double modification of biomolecules via a bioorthogonal reaction. The approach is based on the use of dichlorotetrazine as a trifunctional platform. The first two functionalizations of dichlorotetrazine were performed by successive nucleophilic substitution of the two chlorine atoms of the platform. The choice of the nucleophiles involved in these two reactions is essential and, by choosing an appropriate combination of nucleophiles (i.e. the use of an amine for the first step and a thiol for the second one), we were able to synthesize a series of doubly-substituted tetrazines with groups for the chelation of radiometals on one hand, and with organic fluorophores on the other hand. One of the bifunctionalized tetrazines was then chemoselectively conjugated to an antibody modified with a bicyclo[6.1.0]nonyne group via the IEDDA reaction. This approach has been exemplified by the development of a targeted bimodal nuclear/optical imaging agent. The preclinical evaluation of this bioconjugate demonstrated adequate pharmacokinetics, allowing in vivo diagnostic images to be recorded.

During this work, the introduction of the click function, in this case the bicyclo[6.1.0]nonyne, on the protein was carried out using a random amine labeling step. In order to fully benefit from the advantages of the specificity of bioconjugation, it would be important to apply this strategy to a biomolecule whose click reactive group has been introduced by genetic engineering or any other regioselective methods. In this way, the number and position of the different grafted groups could be completely controlled.

The advantage of this approach over the different ternary platforms reported in the literature is its simplicity. ${ }^{38,39}$ Indeed, the regiospecific conjugation step on the biomolecule is carried out by the platform itself, and the number of steps required to obtain the desired compound is therefore considerably reduced. The simplicity of the synthesis makes this approach highly versatile, allowing to introduce independently one from the other any macrocyclic chelator or fluorescent dye (such as the clinically validated IRDye800 CW fluorophore), in order to finely tune the properties of the resulting imaging agent. Bimodal molecular imaging is an obvious application to this innovative bioconjugation strategy. However, it should be kept in mind that the modularity of this approach makes it applicable to the many areas requiring the double regioselective modification of proteins. For example, in the field of targeted therapy, it could facilitate the development of theranostic or bivalent cytotoxic agents. ${ }^{8}$ 


\section{EXPERIMENTAL PROCEDURES}

Synthetic details. For detailed synthesis procedures and characterization of the precursors and bimodal probes 1-9, see Supplementary Information. For RP-HPLC-MS, HRMS, ${ }^{1} \mathrm{H}$ NMR and ${ }^{13} \mathrm{C}$ NMR spectra of the compounds in this article, see the Supplementary Figs. S2-S29. The synthesis of the bimodal probe 7 and its conjugation to trastuzumab are detailed below.

\section{Synthesis of the bimodal probe 7 .}

2,2',2"-(10-(4-((2-aminoethyl)amino)-1-carboxy-4-oxobutyl)-1,4,7,10-tetraazacyclododecane1,4,7-triyl)triacetic acid (DOTAGA- $\left.\mathrm{NH}_{2} \cdot 1.7 \mathrm{H}_{2} \mathrm{O}\right)(243 \mathrm{mg}, 443 \mu \mathrm{mol})$ was dissolved in borate buffer $(2.5 \mathrm{M}, \mathrm{pH} 8)(7 \mathrm{~mL})$ and the $\mathrm{pH}$ was adjusted to $\mathrm{pH} 8$ with $\mathrm{NaOH} 2.5 \mathrm{M}$. Then $s$ dichlorotetrazine $(68 \mathrm{mg}, 443 \mu \mathrm{mol}, 1.0 \mathrm{eq})$ in $\mathrm{MeCN}(2 \mathrm{~mL})$ was added. The reaction was stopped after $1 \mathrm{~h} 45$ at room temperature and solvents were eliminated under reduced pressure. Purification by semi-preparative RP-HPLC on a BetaBasic-18 column (eluents: $\mathrm{H}_{2} \mathrm{O} 0.1 \%$ TFA, MeCN 0.1\% TFA) afforded DOTAGA-NH-Tz-Cl-1.5TFA 1 as an orange powder (154 mg, 17\%, purity: >99\%). ${ }^{1} \mathrm{H}$ NMR (500 MHz, $\left.\mathrm{D}_{2} \mathrm{O}\right) \partial 2.01\left(\mathrm{~m}, 2 \mathrm{H} ; \mathrm{CH}_{2}\right), 2.55\left(\mathrm{~m}, 2 \mathrm{H} ; \mathrm{CH}_{2}\right)$, 2.96 to 3.50 (m, 12H; $\mathrm{CH}_{2}$ ), 3.51 (m, $\left.2 \mathrm{H} ; \mathrm{CH}_{2}\right), 3.53$ to 3.68 (m, $\left.4 \mathrm{H} ; \mathrm{CH}_{2}\right), 3.71\left(\mathrm{~m}, 2 \mathrm{H} ; \mathrm{CH}_{2}\right.$ ), 3.73 to 4.29 (m, 7H; $\left.\mathrm{CH}, \mathrm{CH}_{2}\right) ;{ }^{13} \mathrm{C}$ NMR $\left(125.7 \mathrm{MHz}, \mathrm{D}_{2} \mathrm{O}\right) \partial 32.9,38.3,40.2,42.2,44.0$ to 64.0, 116.3 (q, ${ }^{1} \mathrm{~J}=290.0 \mathrm{~Hz}, \mathrm{TFA}$ ), 162.8 (poorly resolved, TFA) 158.5, 161.6, 166.0 to 176.0; MS: $\mathrm{C}_{19} \mathrm{H}_{31} \mathrm{ClN}_{9} \mathrm{O}_{7}{ }^{+}\left([\mathrm{M}+\mathrm{H}]^{+}\right) \mathrm{m} / \mathrm{z}$ calculated: 633.3 , found: 633.2 .

DOTAGA-NH-Tz-Cl-1.5TFA $1(5.7 \mathrm{mg}, 7.1 \mu \mathrm{mol})$ and sulfonated Cyanine 5.0-SH (Supporting Information; $5.4 \mathrm{mg}, 7.54 \mu \mathrm{mol}, 1.1 \mathrm{eq}$ ) were dissolved in anhydrous DMF $(800 \mu \mathrm{L})$ and DIPEA $(8.47 \mu \mathrm{L}, 45.3 \mu \mathrm{mol}, 6.4 \mathrm{eq})$ was added. After $5 \mathrm{~h}$ at $75^{\circ} \mathrm{C}$, the solvent was eliminated under reduced pressure. Purification by semi-preparative RP-HPLC (eluents: $\mathrm{H}_{2} \mathrm{O}$ 0.1\% FA, MeCN 0.1\% FA) gave DOTAGA-NH-Tz-S-sulfonated Cyanine 5.07 as a blue powder (3.8 mg, 34\%, purity: 82\%). ${ }^{1} \mathrm{H}$ NMR (500 MHz, D $\left.2 \mathrm{O}\right) \partial 1.31\left(\mathrm{~m}, 5 \mathrm{H} ; \mathrm{CH}_{2}, \mathrm{CH}_{3}\right), 1.58$ (m, 2H; $\mathrm{CH}_{2}$ ), 1.61 (s, 6H; $\left.\mathrm{CH}_{3}\right), 1.67$ (s, 6H; $\left.\mathrm{CH}_{3}\right), 1.81$ (m, 2H; $\left.\mathrm{CH}_{2}\right), 1.88\left(\mathrm{~m}, 2 \mathrm{H} ; \mathrm{CH}_{2}\right)$, $2.20\left(\mathrm{t},{ }^{3} \mathrm{~J}=6.6 \mathrm{~Hz}, 2 \mathrm{H} ; \mathrm{CH}_{2}\right), 2.44\left(\mathrm{~m}, 2 \mathrm{H} ; \mathrm{CH}_{2}\right), 2.97$ to $3.63\left(\mathrm{~m}, 26 \mathrm{H} ; \mathrm{CH}_{2}\right), 3.82(\mathrm{~m}, 5 \mathrm{H}$; $\left.\mathrm{CH}_{2}, \mathrm{CH}\right), 4.07$ (m, 4H; $\left.\mathrm{CH}_{2}\right), 6.15\left(\mathrm{~d},{ }^{3} \mathrm{~J}=13.6 \mathrm{~Hz}, 1 \mathrm{H} ; \mathrm{CH}\right), 6.23\left(\mathrm{~d},{ }^{3} \mathrm{~J}=13.6 \mathrm{~Hz}, 1 \mathrm{H} ; \mathrm{CH}\right)$, $6.48\left(\mathrm{t},{ }^{3} \mathrm{~J}=12.3 \mathrm{~Hz}, 1 \mathrm{H} ; \mathrm{CH}\right), 7.33\left(\mathrm{~d},{ }^{3} \mathrm{~J}=8.0 \mathrm{~Hz}, 2 \mathrm{H} ; \mathrm{CH}\right), 7.79$ to $7.88(\mathrm{~m}, 4 \mathrm{H} ; \mathrm{CH}), 7.96$ $(\mathrm{m}, 1 \mathrm{H} ; \mathrm{CH}), 8.01(\mathrm{~m}, 1 \mathrm{H} ; \mathrm{CH})$; The low amount of product available did not allow us to perform proper ${ }^{13} \mathrm{C}$ NMR; HRMS: $\mathrm{C}_{58} \mathrm{H}_{82} \mathrm{~N}_{13} \mathrm{O}_{16} \mathrm{~S}_{3}{ }^{+}\left([\mathrm{M}+\mathrm{H}]^{+}\right) \mathrm{m} / \mathrm{z}$ calculated: 1312.51591, found 1312.51945 . 
Conjugation of the bimodal probe 7 to trastuzumab-BCN 9. $75.3 \mu \mathrm{L}$ of a $5 \mathrm{mM}$ stock solution of bimodal probe 7 in $\mathrm{H}_{2} \mathrm{O}(376.6 \mathrm{nmol}, 33.0 \mathrm{eq})$ was added to a $10 \mathrm{mg} / \mathrm{mL}$ solution of trastuzumab-BCN 9 (1.71 mg, $11.4 \mathrm{nmol})$ in PBS (1 M, pH 7.4) with 10\% of DMSO. The solution was stirred in a thermomixer $\left(800 \mathrm{rpm}, 37^{\circ} \mathrm{C}\right)$ during $16 \mathrm{~h}$. Then, excess of 7 was removed by FPLC (Äkta Pure 25 M chromatography system, GE Healthcare Life Sciences, USA) on a Hitrap desalting column (Sephadex G-25 Superfine resin, cross-linked dextran, bead size $15-70 \mu \mathrm{m}, 16 \times 25 \mathrm{~mm}, 5 \mathrm{~mL})$ with $\mathrm{AcONH}_{4}(0.1 \mathrm{M}, \mathrm{pH} 5.8)$ as eluent. The pure, dually labeled, trastuzumab 10 was obtained as a $2.0 \mathrm{mg} / \mathrm{mL}$ solution in $\mathrm{AcONH}_{4}$ (protein recovery = $49 \%$ ). Based on the spectroscopy measurements, the degree of labeling (amount of bimodal probe per antibody) was determined to be 1.4 .

A correction factor (0.05) was introduced to account for the contribution of the fluorophore absorbance at $280 \mathrm{~nm}$. The molar concentration of the fluorophore was calculated by UV spectrophotometry by using the extinction coefficient of the sulfonated Cyanine 5.0 in PBS (250000 L.mol $\left.{ }^{-1} \cdot \mathrm{cm}^{-1}\right)$.

Characterization of the conjugate 10. Matrix-assisted laser desorption ionization/time of flight (MALDI/TOF) mass spectra (Supplementary Fig. 31-33) were recorded on a Bruker Daltonics Ultraflex II LRF 2000 mass spectrometer using a matrix made of saturated solution of sinapinic acid (Sigma-Aldrich) in $\mathrm{MeCN} / \mathrm{H}_{2} \mathrm{O}(60: 40, v / v)$ with TFA $0.1 \%$. The target used is an MTP AnchorChip ${ }^{\text {TM }} 384$ (Bruker).

$\mathrm{UV}-\mathrm{Vis}$ absorbance and fluorescence spectra of $\mathbf{1 0}$ in $\mathrm{AcONH}_{4}$ buffer (0.1 M, pH 5.8) were recorded on a CLARIOstar microplate reader (BMG Labtech, USA). The excitation spectrum was measured from $320 \mathrm{~nm}$ to $670 \mathrm{~nm}$ with a fixed emission wavelength at $700 \mathrm{~nm}$. The emission spectrum was measured from $630 \mathrm{~nm}$ to $740 \mathrm{~nm}$ with a fixed excitation wavelength at $600 \mathrm{~nm}$. Values for $\lambda_{\mathrm{Abs}, \max }, \lambda_{\mathrm{Ex}, \max }$ and $\lambda_{\mathrm{Em}, \max }$ were respectively $651 \mathrm{~nm}, 655 \mathrm{~nm}$ and $673 \mathrm{~nm}$.

Stability in serum of conjugate 10 by gel electrophoresis. $10.53 \mu \mathrm{L}$ of a stock solution of 10 at $1.9 \mathrm{mg} / \mathrm{mL}(20 \mu \mathrm{g})$ in $\mathrm{AcONH}_{4}$ buffer $(0.1 \mathrm{M}, \mathrm{pH} 5.8)$ were stirred in a thermomixer (800 rpm, $37^{\circ} \mathrm{C}$, dark) with $20 \mu \mathrm{L}$ of human plasma and $9.47 \mu \mathrm{L}$ of water. The final concentration in 10 was $0.5 \mathrm{mg} / \mathrm{mL}$. After 1, 2, 4, 8, 24 or $48 \mathrm{~h}$, the reactions were stopped and $5 \mu \mathrm{L}$ samples were analyzed by sodium dodecyl sulfate polyacrylamide gel electrophoresis (SDS-PAGE) analysis. The samples were not denaturated at $90{ }^{\circ} \mathrm{C}$ prior to the electrophoresis to avoid unsuitable degradation. SDS-PAGE analyses were performed on Mini-PROTEAN® 
$\mathrm{TGX}^{\mathrm{TM}} 4$ to $20 \%$ Precast gel (BioRad) in non-reductive conditions. $5 \mu \mathrm{L}$ of sample solution at a concentration of about $1 \mathrm{mg} / \mathrm{mL}$ was mixed with $5 \mu \mathrm{L}$ of loading. The loading buffer was constituted of $0.125 \mathrm{M}$ Tris $\mathrm{HCl}, 20 \%$ glycerol, $4 \%$ SDS and $0.004 \%$ bromophenol blue. After loading, the electrophoresis was started $(160 \mathrm{~V}, 45 \mathrm{~min})$ in a running buffer constituted of $0.3 \%$ Tris $\mathrm{HCl}, 1.92 \mathrm{M}$ glycine, $0.1 \% \mathrm{SDS}$ and $0.05 \%$ sodium azide. The protein ladder used as reference was a PageRuler ${ }^{\mathrm{TM}}$ Plus Prestained (Thermo Scientific, USA). For visualization of the fluorescent sulfonated Cyanine 5.0-conjugate, a fluorescence image of the gel was recorded on an IVIS Lumina III In Vivo Imaging System (Perkin Elmer) in pair filter mode 620/670 nm. Bandwidth was of $20 \mathrm{~nm}$ for excitation and $40 \mathrm{~nm}$ for emission. Then, the gel was stained with Coomassie brillant blue during $1 \mathrm{~h}$ (see Supporting Fig. S35).

Radiolabeling of the conjugate 10. The radiolabeling was performed by using $225 \mathrm{MBq}$ of ${ }^{111} \mathrm{InCl}_{3}$ and $375.0 \mu \mathrm{g}$ of $\mathbf{1 0}$. The solution was stirred for $1 \mathrm{~h}$ at $37^{\circ} \mathrm{C}$ and the reaction monitored by iTLC. $51.5 \mu \mathrm{L}$ of $50 \mathrm{mM}$ EDTA in $0.1 \mathrm{M}$ ammonium acetate was added to the reaction mixture. The resulting ${ }^{111}$ In-EDTA was then removed by ultrafiltration on an Amicon Ultra Ultracel-30 kDa (Merck Millipore, USA) and iTLC was performed, that showed a radiochemical purity $>99 \%$. ITLCs performed before and after ultrafiltration gave a ratio of ${ }^{111}$ In complexed of $58 \%$ and a radiochemical purity of $99 \%$, respectively. The measure of radioactivity introduced in the Amicon Ultra Ultracel-30 $\mathrm{kDa}$ and obtained after the ultrafiltration allowed us to calculate a purification yield of $85 \%$, a global ratio of recovered protein of $82 \%$ and a specific activity of $366 \mathrm{MBq} / \mathrm{mg}$. After purification, the radiolabeled antibody 11 was diluted in PBS 0.1 M, pH 7.4, for injection.

Cell and animal models. BT-474 cells (ATCC, Rockville, MD) were grown as adherent monolayer in RPMI-1640 medium (Lonza, Belgium) supplemented with 10\% fetal calf serum (Lonza, Belgium). Cancer cells were harvested just prior to experiments and were washed twice with RPMI1640 medium and resuspended in a mixture of RPMI1640 and matrigel (BD Biosciences, USA) $(1 / 1, v / v)$. The viability of the cells was assessed by trypan blue exclusion assay. In all cases, viability was greater than $90 \%$.

All animal studies were conducted in accordance with the legislation on the use of laboratory animals (directive 2010/63/EU) and were approved by accredited Ethical committee (C2ea Grand Campus $n^{\circ} 105$ ) and the French Ministries of Research (project \#7830) and Agriculture (A 21231016 EA). BALB/c nude mice ( $n=7 ; 6-8$ weeks old, purchased from Charles River, 
France) were subcutaneously injected in the flank with $2 \times 10^{7}$ BT-474 human breast carcinoma cells. After tumor growth, mice were randomized into 2 groups.

SPECT-CT/fluorescence imaging. A first group of mice $(n=4)$ was injected with the ${ }^{111}$ In radiolabeled bioconjugate $\mathbf{1 1}$ intravenously in the lateral tail vein. The injected doses per mice were 22.4-24.7 $\mu \mathrm{g}(8.2-9.0 \mathrm{MBq})$ in solution in PBS $(100 \mu \mathrm{L})$. The second group $(\mathrm{n}=3)$ was co-injected with 16.1-20.9 $\mu \mathrm{g}$ (5.9-7.6 MBq) of ${ }^{111}$ In radiolabeled bioconjugate and an excess of trastuzumab (100 equivalents) per mice. For all imaging procedures, mice were anesthetized under isoflurane (1.5-2.5\% in oxygen).

SPECT-CT imaging was performed $24 \mathrm{~h}$ after the injection of the radiolabeled conjugate using a NanoSPECT-CT small animal imaging tomographic $\gamma$-camera (Mediso, Hungary). CT acquisitions (45 kV, $500 \mathrm{~ms}$ ) were first acquired during 4-6 min, followed by helical SPECT acquisitions with 30-40 s per projection frame resulting in acquisition times of 15-25 min. Both indium-111 photopeaks (171 and $245 \mathrm{keV}$ ) were used with $10 \%$ wide energy windows. The CT and SPECT reconstructions were performed using image processing softwares provided by Invicro. The SPECT-CT fusion image was obtained using the InVivoScope software (Invivro, USA).

Immediately after the end of SPECT-CT imaging, the animals were transferred to the optical imager. The fluorescence signal was monitored using the Perkin Elmer Ivis Lumina III system with the following excitation/emission settings: $620 \mathrm{~nm} / 670 \mathrm{~nm}$. Data were analyzed with the Living Image ${ }^{\circledR}$ software. Values are reported as radiant efficiencies, allowing direct comparison of images from each experiment.

Biodistribution. After optical imaging, mice were euthanized and organs collected for ex vivo optical imaging at $24 \mathrm{~h}$ post-injection (Supplementary Fig. S39). Fluorescence intensities were measured and normalized to photon per second per $\mathrm{cm}^{2}$ using the region of interest (ROI) function of the Living Image $\AA$ software. The ROI covers the entire tissue. Then, the radioactivity of each organ was measured with a scintillation $\gamma$-counter (Wizard 1480, Perkin Elmer, USA). Data were then converted to percentage of injected dose and to percentage of injected dose per gram of tissue (the injected doses being corrected for subcutaneously injected material remaining in the animal tail and for radioactive decay). 
Statistics. All statistical analyses were performed using GraphPad Prism 6 or 7 (GraphPad Software, USA). All data were obtained at least in triplicate and results reported as mean \pm standard deviation. 


\section{ACKNOWLEDGEMENTS}

Financial support was provided by the French National Research Agency (ANR) under the programs Investissements d'Avenir (ANR-10-EQPX-05-01/IMAPPI Equipex) and AAP Générique 2017 (project ZINELABEL), the CNRS and the Université de Bourgogne. C.C. was funded by the Ministry of Higher Education, Research and Innovation. This work is also part of the project Pharmaco-imagerie et agents théranostiques supported by the Conseil Régional de Bourgogne Franche-Comté through the Plan d'Action Régional pour l'Innovation (PARI) and by the European Union through the PO FEDER-FSE 2014/2020 Bourgogne program. This work was performed within Pharm'image, a regional centre of excellence in Pharmaco-imaging and within the GDR CNRS 2037 AIM (Molecular Imaging Agents). The authors thank the "Plateforme d'Analyse Chimique et de Synthèse Moléculaire de l'Université de Bourgogne" (http://www.wpcm.fr) for access to analytical instrumentation and Prof. Anthony Romieu for kindly giving us the disulfonated Cyanine 5.0 dye.

\section{SUPPORTING INFORMATION AVAILABLE}

Synthesis and characterization of compounds 1-11. In vitro stability data of compounds 7 an 10. Ex vivo biodistribution data of compound 11.

\section{AUTHOR CONTRIBUTIONS}

V.G. designed the project. C.C. synthesized the precursors, multimodal probes, and conjugates, and characterized all compounds. M.M. assisted C.C. in performing the radiolabeling of the conjugates for in vivo experiments, and analyzed the data from SPECT-CT. J.M.V. assisted in performing SPECT-CT experiments. P.S.B and B.C. developed the tumor model and assisted in the design of the in vivo experiment. C.C. and V.G. wrote the manuscript. F.D. helped critically appraise and edit the manuscript. All authors reviewed the final manuscript.

\section{CONFLICT OF INTEREST DISCLOSURE}

C.C., F.D., and V.G. are inventors on a patent application that describes the use of tetrazine derivatives for the modification of biomolecules (PCT/EP2018/057520). 


\section{REFERENCES}

(1) Srinivasarao, M., Galliford, C. V., and Low, P. S. (2015) Principles in the design of ligandtargeted cancer therapeutics and imaging agents. Nat. Rev. Drug Discov. 14, 203-219.

(2) Wu, M., and Shu, J. (2018) Multimodal Molecular Imaging: Current Status and Future Directions. Contrast Media Mol. Imaging 2018.

(3) Hekman, M. C. H., Boerman, O. C., Weijert, M. de, Bos, D. L., Oosterwijk, E., Langenhuijsen, H. F., Mulders, P. F. A., and Rijpkema, M. (2016) Targeted Dual-Modality Imaging in Renal Cell Carcinoma: An Ex Vivo Kidney Perfusion Study. Clin. Cancer Res. 22, $4634-4642$.

(4) Zhang, H., Desai, P., Koike, Y., Houghton, J., Carlin, S., Tandon, N., Touijer, K., and Weber, W. A. (2017) Dual-Modality Imaging of Prostate Cancer with a Fluorescent and Radiogallium-Labeled Gastrin-Releasing Peptide Receptor Antagonist. J. Nucl. Med. 58, 2935 .

(5) Lütje, S., Rijpkema, M., Franssen, G. M., Fracasso, G., Helfrich, W., Eek, A., Oyen, W. J., Colombatti, M., and Boerman, O. C. (2014) Dual-Modality Image-Guided Surgery of Prostate Cancer with a Radiolabeled Fluorescent Anti-PSMA Monoclonal Antibody. J. Nucl. Med. 55, 995-1001.

(6) Bunschoten, A., Buckle, T., Visser, N. L., Kuil, J., Yuan, H., Josephson, L., Vahrmeijer, A. L., and van Leeuwen, F. W. B. (2012) Multimodal interventional molecular imaging of tumor margins and distant metastases by targeting $\alpha \mathrm{v} \beta 3$ integrin. Chembiochem 13, 1039-1045.

(7) Povoski, S. P., Neff, R. L., Mojzisik, C. M., O’Malley, D. M., Hinkle, G. H., Hall, N. C., Murrey, D. A., Knopp, M. V., and Martin, E. W. (2009) A comprehensive overview of radioguided surgery using gamma detection probe technology. World J. Surg. Oncol. 7, 11.

(8) Maruani, A., Richards, D. A., and Chudasama, V. (2016) Dual modification of biomolecules. Org. Biomol. Chem. 14, 6165-6178.

(9) Zhao, J., Chen, J., Ma, S., Liu, Q., Huang, L., Chen, X., Lou, K., and Wang, W. (2018) Recent developments in multimodality fluorescence imaging probes. Acta Pharm. Sin B. 8, $320-338$.

(10) Guo, J., Kumar, S., Prashad, A., Starkey, J., and Singh, S. K. (2014) Assessment of physical stability of an antibody drug conjugate by higher order structure analysis: impact of thiol- maleimide chemistry. Pharm. Res. 31, 1710-1723.

(11) Rijpkema, M., Bos, D. L., Cornelissen, A. S., Franssen, G. M., Goldenberg, D. M., Oyen, W. J., and Boerman, O. C. (2015) Optimization of Dual-Labeled Antibodies for Targeted 
Intraoperative Imaging of Tumors. Mol. Imaging 14, 348-355.

(12) Adumeau, P., Sharma, S. K., Brent, C., and Zeglis, B. M. (2016) Site-Specifically Labeled Immunoconjugates for Molecular Imaging--Part 1: Cysteine Residues and Glycans. Mol. Imaging Biol. 18, 1-17.

(13) Adumeau, P., Sharma, S. K., Brent, C., and Zeglis, B. M. (2016) Site-Specifically Labeled Immunoconjugates for Molecular Imaging--Part 2: Peptide Tags and Unnatural Amino Acids. Mol. Imaging Biol. 18, 153-165.

(14) Hu, Q.-Y., Berti, F., and Adamo, R. (2016) Towards the next generation of biomedicines by site-selective conjugation. Chem. Soc. Rev. 45, 1691-1719.

(15) Krall, N., da Cruz, F. P., Boutureira, O., and Bernardes, G. J. L. (2016) Site-selective protein-modification chemistry for basic biology and drug development. Nat. Chem. 8, 103113.

(16) Lang, K., Davis, L., Wallace, S., Mahesh, M., Cox, D. J., Blackman, M. L., Fox, J. M., and Chin, J. W. (2012) Genetic Encoding of bicyclononynes and trans-cyclooctenes for sitespecific protein labeling in vitro and in live mammalian cells via rapid fluorogenic Diels-Alder reactions. J. Am. Chem. Soc. 134, 10317-10320.

(17) Lang, K., and Chin, J. W. (2014) Cellular incorporation of unnatural amino acids and bioorthogonal labeling of proteins. Chem. Rev. 114, 4764-4806.

(18) Mayer, S., and Lang, K. (2017) Tetrazines in Inverse-Electron-Demand Diels-Alder Cycloadditions and Their Use in Biology. Synthesis 49, 830-848.

(19) Oliveira, B. L., Guo, Z., and Bernardes, G. J. L. (2017) Inverse electron demand DielsAlder reactions in chemical biology. Chem. Soc. Rev. 46, 4895-4950.

(20) Blackman, M. L., Royzen, M., and Fox, J. M. (2008) Tetrazine Ligation: Fast Bioconjugation Based on Inverse-Electron-Demand Diels-Alder Reactivity. J. Am. Chem. Soc. 130, 13518-13519.

(21) Devaraj, N. K., Weissleder, R., and Hilderbrand, S. A. (2008) Tetrazine-based cycloadditions: application to pretargeted live cell imaging. Bioconjugate Chem. 19, $2297-$ 2299.

(22) Wu, H., and Devaraj, N. K. (2015) Inverse Electron-Demand Diels-Alder Bioorthogonal Reactions. Top. Curr. Chem. (Z) 374, 3.

(23) Meimetis, L. G., Boros, E., Carlson, J. C., Ran, C., Caravan, P., and Weissleder, R. (2016) Bioorthogonal Fluorophore Linked DFO-Technology Enabling Facile Chelator Quantification and Multimodal Imaging of Antibodies. Bioconjugate Chem. 27, 257-263.

(24) Novák, Z., Bostai, B., Csékei, M., Lorincz, K., and Kotschy, A. (2003) Selective 
nucleophilic substitutions on tetrazines. Heterocycles 60, 2653-2668.

(25) Clavier, G., and Audebert, P. (2010) s-Tetrazines as building blocks for new functional molecules and molecular materials. Chem. Rev. 110, 3299-3314.

(26) Venkateswara Rao, B., Dhokale, S., Rajamohanan, P. R., and Hotha, S. (2013) A tetrazine templated method for the synthesis of ternary conjugates. Chem. Commun. (Camb.) 49, 1080810810.

(27) Zhu, J., Li, S., Wängler, C., Wängler, B., Lennox, R. B., and Schirrmacher, R. (2015) Synthesis of 3-chloro-6-((4-(di-tert-butyl[(18)F]fluorosilyl)-benzyl)oxy)-1,2,4,5-tetrazine ([(18)F]SiFA-OTz) for rapid tetrazine-based (18)F-radiolabeling. Chem. Commun. (Camb.) 51, $12415-12418$.

(28) Wu, D., and O'Shea, D. F. (2017) Fluorogenic NIR-probes based on 1,2,4,5-tetrazine substituted BF2-azadipyrromethenes. Chem. Commun. (Camb.) 53, 10804-10807.

(29) Canovas, C., Moreau, M., Bernhard, C., Oudot, A., Guillemin, M., Denat, F., and Goncalves, V. (2018) Site-Specific Dual Labeling of Proteins on Cysteine Residues with Chlorotetrazines. Angew. Chem. Int. Ed. 57, 10646-10650.

(30) Price, E. W., and Orvig, C. (2014) Matching chelators to radiometals for radiopharmaceuticals. Chem. Soc. Rev. 43, 260-290.

(31) Fani, M., Del Pozzo, L., Abiraj, K., Mansi, R., Tamma, M. L., Cescato, R., Waser, B., Weber, W. A., Reubi, J. C., and Maecke, H. R. (2011) PET of somatostatin receptor-positive tumors using $64 \mathrm{Cu}$ - and $68 \mathrm{Ga}$-somatostatin antagonists: the chelate makes the difference. $J$. Nucl. Med. 52, 1110-1118.

(32) Ghosh, S. C., Pinkston, K. L., Robinson, H., Harvey, B. R., Wilganowski, N., Gore, K., Sevick-Muraca, E. M., and Azhdarinia, A. (2015) Comparison of DOTA and NODAGA as chelators for (64)Cu-labeled immunoconjugates. Nucl. Med. Biol. 42, 177-183.

(33) Moreau, M., Raguin, O., Vrigneaud, J.-M., Collin, B., Bernhard, C., Tizon, X., Boschetti, F., Duchamp, O., Brunotte, F., and Denat, F. (2012) DOTAGA-trastuzumab. A new antibody conjugate targeting HER2/Neu antigen for diagnostic purposes. Bioconjugate Chem. 23, 11811188.

(34) Boonstra, M. C., de Geus, S. W. L., Prevoo, H. A. J. M., Hawinkels, L. J. A. C., van de Velde, C. J. H., Kuppen, P. J. K., Vahrmeijer, A. L., and Sier, C. F. M. (2016) Selecting Targets for Tumor Imaging: An Overview of Cancer-Associated Membrane Proteins. Biomark. Cancer 8, 119-133.

(35) Kim, C. H., Axup, J. Y., Dubrovska, A., Kazane, S. A., Hutchins, B. A., Wold, E. D., Smider, V. V., and Schultz, P. G. (2012) Synthesis of Bispecific Antibodies using Genetically 
Encoded Unnatural Amino Acids. J. Am. Chem. Soc. 134, 9918-9921.

(36) Rahim, M. K., Kota, R., and Haun, J. B. (2015) Enhancing reactivity for bioorthogonal pretargeting by unmasking antibody-conjugated trans-cyclooctenes. Bioconjugate Chem. 26, $352-360$.

(37) Zhou, Y., Kim, Y.-S., Milenic, D. E., Baidoo, K. E., and Brechbiel, M. W. (2014) In vitro and in vivo analysis of indocyanine green-labeled panitumumab for optical imaging-a cautionary tale. Bioconjugate Chem. 25, 1801-1810.

(38) Clavé, G., Volland, H., Flaender, M., Gasparutto, D., Romieu, A., and Renard, P.-Y. (2010) A universal and ready-to-use heterotrifunctional cross-linking reagent for facile synthetic access to sophisticated bioconjugates. Org. Biomol. Chem. 8, 4329-4345.

(39) Zong, H., Goonewardena, S. N., Chang, H.-N., Otis, J. B., and Baker, J. R. (2014) Sequential and parallel dual labeling of nanoparticles using click chemistry. Bioorg. Med. Chem. 22, 6288-6296. 
TABLE OF CONTENTS GRAPHIC
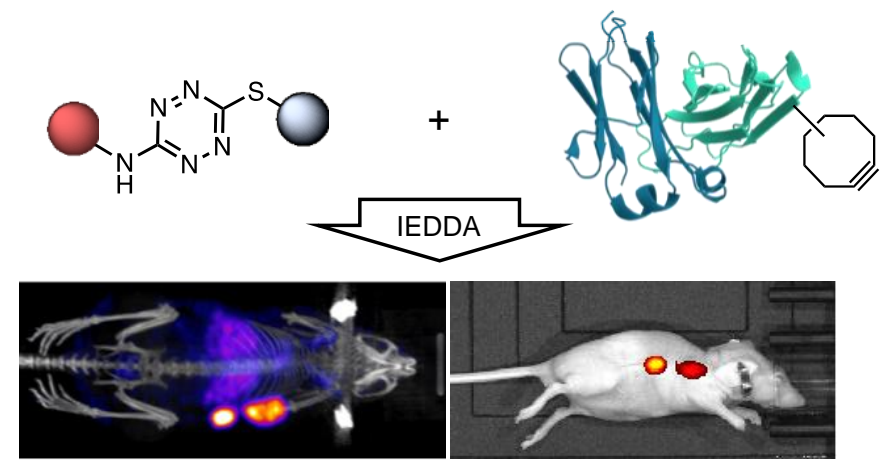

DOUBLY-LABELED PROTEINS FOR MULTIMODAL IMAGING 